\title{
Physical Activity is a Preventive Factor Against SARS- COV-2 in Healthy Subjects (Possible Cellular and Molecular Mechanisms)
}

\author{
Mehdi Kushkestani $^{1}$, Mohsen Parvani ${ }^{2 *}$ and Ana maria Teixeira ${ }^{3}$ \\ ${ }^{1} 1$ M.Sc. Student of Exercise Physiology, Faculty of Physical Education and Sport Sciences, AllamehTabataba'i University, Tehran, Iran \\ ${ }^{2}$ M.Sc. Student of Exercise Physiology, Faculty of Physical Education and Sport Sciences, AllamehTabataba'i University, Tehran, Iran \\ ${ }^{3}$ Associated Professor at the Faculty of Sports Science and Physical Education and coordinator of the PhD Course in Sports Science, \\ University of Coimbra, Coimbra, Portugal
}

*Corresponding author: Mohsen Parvani, M.Sc. Student of Exercise Physiology, Faculty of Physical Education and Sport Sciences,

AllamehTabataba'i University, Tehran, Iran

\section{ARTICLE INFO}

Received: 蔧 August 02, 2020

Published: 幽 August 10, 2020

Citation: Mehdi Kushkestani, Mohsen Parvani, Ana maria Teixeirac. Physical Activity is a Preventive Factor Against SARS-COV-2 in Healthy Subjects (Possible Cellular and Molecular Mechanisms). Biomed J Sci \& Tech Res 29(3)-2020. BJSTR. MS.ID.004799.

Keywords: Aerobic Training; Exercise; Immunology; Resistance Training; SARSCOV2

\section{ABSTRACT}

SARS-COV-2 is a new type of coronavirus that was first detected in China in December 2019 and is now known as a pandemic. The human immune system responds to this viral infection with a set of defence mechanisms. On the other hand, given the complex process of vaccine production, it seems that the prevention of SARS-COV-2 infection and strengthening the immune system is the first line fights the high prevalence of this infection. In this regard, exercise is considered as one of the strategies that can have the effects of immunoenhancing and immunosuppression and reduce or increase the risk of infection. In this review article, we intend to make suggestions based on the cellular and molecular responses of the immune system to SARS-COV-2 infection and the effects of different types of exercise on the immune system and the risk of infection, to reduce the risk of infection and possibly preventing SARS-COV-2 offer.

Abbreviations: WHO: World Health Organization, IgG: Immunoglobin G; NK Cells: Natural Killer Cells, Th1: T Helper1; Th2: T Helper2;sIgA: Salivary Immunoglobin A; mRNA: Messenger RNA, PAMPs: Pathogen-Associated Molecular Patterns; +ssRNA: Positive Sense Single-Stranded RNA, TLR: Toll-Like Receptor, RIG: Retinoid-Inducible Gene, MDA5: Melanoma Differentiation-Associated Gene 5; NF- $\kappa B$ : Nuclear FactorkB; AP-1: Activator Protein 1; IRF: Interferon Response Factor; TNFa: Tumor Necrosis Factor a; IL: Interleukin, CCL2: C-C Motif Chemokine Ligand 2; CXCL8: C-X-C Motif Chemokine Ligand 8; IFN: Interferon; ISGs: Interferon Stimulating Genes, ACSM: American College of Sports Medicine, VO2max: Maximum Rate of Oxygen Consumption, HR: Heart Rate, MHR: Maximum Heart Rate, RPE: Rating of Perceived Exertion, TGF- $\beta$ : Transforming Growth Factor $\beta$, GSH: Glutathione; URTI: Upper Respiratory Tract Infection; 1RM: 1-Repetition Maximum

\section{Introduction}

The SARS-COV-2 is a new corona viral strain responsible for the December 2019 pandemic. The Coronavirus disease-2019 was stated to be caused by a new strain of SARS-CoV-2 in December 2019 [1]. Cases have shown pneumonia-like signs and others related to critical respiratory syndrome [2]. According to World
Health Organization (WHO), the huge expanse of SARS-COV-2 has reached almost all countries in the world. As of 26 July 2020, there have been 15785641 reported cases for SARS-COV-2 out of which 640016 mortality [3]. The viral infections are commonly related to higher respiratory tract infections, of which the signs and symptoms 
generally involve fever, headache, and cough; some subjects may have lower respiratory tract infections. In contrast, SARS-CoV and MERS-CoV infections may remain asymptomatic in the early stage until critical pneumonia, dyspnea, renal insufficiency, and even death. SARS-CoV infection can induce pathological developments such as degeneration, infiltration, and hyperplasia. Damage to the pulmonary interstitial arteriolar walls indicates that inflammatory response plays an important role throughout the course of disease in spites of the pathogenic effect of CoVs. It should be noted that immunopathogenesis is correlated with an immune response out of control, which may appear in pulmonary tissue illness, functional impairment, and lowered lung function.

Chemotactic factors are crucial immune responses toward virus infections, regarding their regulatory effect on dilations and positions of leukocytes in the host lungs. Therefore, spectral changes in chemotactic factors may affect seriously maladjusted immune responses. Immune insufficiency or misdirection can boost viral replication and provoke tissue damages. Also, overactive immune responses may cause immunopathological states [4]. The immune response is vital to control and fire CoV infections, but maladjusted immune responses may result in immunopathology and diminished pulmonary gas exchange. Understanding a deeper comprehension of the interaction between Coronaviruses and the innate and adaptive immune systems of the hosts may shed light on the development and persistence of inflammation in the lungs and probably can decrease the risk of lung inflammation caused by CoVs. Coronaviruses are enveloped, non-segmented, positive-sense single-stranded RNA (+ssRNA) virus genomes in the size ranging from 26 to 32 kilobases. The virion has a nucleocapsid composed of genomic RNA and phosphorylated nucleocapsid (N) protein, which is buried inside phospholipid bilayers and covered by the spike glycoprotein trimmer (S). The membrane (M) protein (a type III transmembrane glycoprotein) and the envelope (E) protein are located among the $\mathrm{S}$ proteins in the virus envelope. SARS-COV-2 belongs to the beta corona virus family [5,6]. A major distinction is seen in the beta subfamily where another (5th) structural protein is observed, the Hemagglutinin esterase protein. The protein is thought to enhance spike protein activity and establish interaction with sialic acids on glycoproteins present on the surface of host cells thereby facilitating viral entry[7](Figure 1).

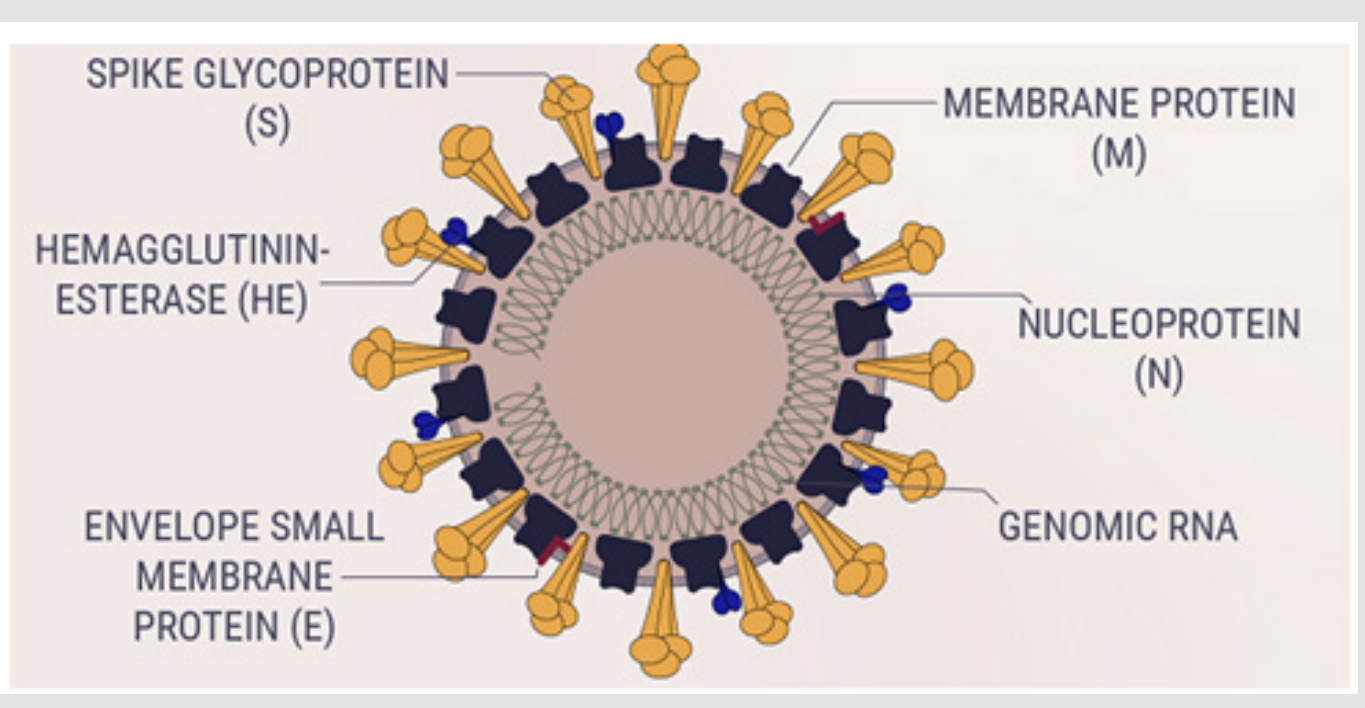

Figure 1: Beta coronavirus family.

To arise an antiviral response, the innate immune system identifies molecular structures that are composed of the invasion of the virus. SARS-COV-2 infection induces immunoglobulin G(IgG) antibodies against $\mathrm{N}$ protein that can be detected by serum as early as day 4 after the onset of disease and with most patients seroconverting by day 14 . Laboratory data of clinical cases revealed that a specific T-cell response against SARS-CoV-2 is necessary for the recognition and killing of infected cells, particularly in the lungs of infected people. now, there is no special antiviral therapy for SARS-COV-2 and the main approaches are supportive[8]. Preventive procedures may efficiently limit the quickly expanding outbreaks of SARS-COV-2, globally [9]. Both cross-sectional and longitudinal studies in humans have confirmed the intense impact that exercise can have on the immune system [10]. Existing investigations have proved that moderate-intensity physical activity has positive enhancing results on immune system responses against viral respiratory infections[11]. Following the moderate-intensity physical activity, an increase in neutrophil and natural killer (NK) cell counts are distinguished and salivary immunoglobin A (sIgA) concentrations are improved [11,12]. Moderate-intensity physical 
activity raises stress hormones, which directs to a decrease in extreme inflammation. This induces an increase in immunity against viral infections via a change in T helper1/T helper2 (Th1/ Th2) cell responses. Twenty to thirty percent decline of upper respiratory tract infections is described in individuals performing moderate levels of physical activity in their regular days [12].

However, there are still numerous uncertainties concerning the response of the immune system to the types of exercise, the intensity and volume of exercise, the response of the immune system throughout recovery, and the acute and chronic responses to exercise. For example, some researchers have determined an increased hazard of infection in response to high-intensity exercise, while some researchers refused this results in their study. Recognizing the most reliable exercise strategies to promote the immune system can diminish the risk of infection and improve the immune system's response after infection. In this review article, we're going to investigate the inflammatory mechanisms involved in SARS-COV-2 and provide the most appropriate type of exercise to improve inflammatory pathways to improve the immune response.

\section{Discussion}

The SARS-COV-2 pandemic has so far reached a highly embarrassing rate[13]. Measures have proceeded to be in place to limit the continued expansion of the virus, and so far, there have been promising outcomes. Though, given that the vaccine and exhaustive treatment for the SARS-COV-2 have not yet been identified, preventive procedures and promote the immune system are the most well-known approaches to diminish the prevalence and develop the immune system's response to this infection. Meanwhile, the role of exercise as one of the saved and cost-effective plans to rise the ability of the immune system upon pathogens (non-communicable and infectious diseases) at different age groups has been proven[10,14]. The modern state of science suggests that the changes in the immune system are dependent on parameters inherent to exercise and that to have all these changes occurring, some cell signalling cascades are occurring, giving rise to a complicated process of phosphorylation/dephosphorylation that culminates in the activation of transcription agents, translation of Messenger RNA's (mRNA's), protein synthesis and cell proliferation[ 15] which can influence the immune system's response to infections. Although, there is currently no examination that investigates exercise mechanisms to promote the immune system's response to prevention and encourage the immune system to combat SARSCOV-2.

\section{Immune System Mechanisms in Response to SARS-COV-2}

Innate Immune Response: To raise an antiviral response, the innate immune system distinguishes molecular compositions that are generated by the invasion of the virus, called pathogenassociated molecular patterns (PAMPs) [16]. For RNA virus such as coronavirus, it is understood that PAMPs found in replicating viruses in the form of viral genomic +ssRNA or double-stranded RNA that are identified by either the endosomal RNA receptors, Toll-like receptor 8 (TLR8), and TLR7 in +SSRNA and the cytosolic RNA sensor, RIG (retinoid-inducible gene)/MDA5 (melanoma differentiation-associated gene 5) $[4,17,18]$. This memory event manages to activation of various signalling pathways and ultimately transcription factors, that is, nuclear factor $\kappa \mathrm{B}(\mathrm{NF}-\kappa \mathrm{B})$, activator protein 1 (AP-1), interferon response factor 3 (IRF3), and IRF7 followed by their nuclear translocation. NF- $\mathrm{BB}$ and AP-1 spur the expression of genes encoding multiple of the molecules required for inflammatory responses, including inflammatory cytokines (eg, tumor necrosis factor [TNFa], interleukin1 [IL1], and IL6) and chemokines (eg, C-C Motif Chemokine Ligand 2 [CCL2], and C-X-C Motif Chemokine Ligand 8 [CXCL8]) 8. TNFa and IL-6 cytokines cooperate with the brain's hypothalamus resulting in physiological changes including an increment in body temperature and fever. Additionally, the response results in vasodilation and enhances vascular permeability. As such, with corresponding fluid accumulation, making enlarged breathing capacities, and by extension breathing trouble 7. IRF3 and IRF7 favour the reproduction of type I interferon (IFN- $\alpha$ and IFN- $\beta$ ), which are critical for antiviral innate immune responses and capable to suppress viral replication and distribution at an early stage [19]. This process can induce complications such as fatigue, debility, and cough in cases [20]. The major purpose is, for SARS-CoV-2, the response to viral infection by type I IFN is suppressed [21]. IFN, in turn, stimulates the JAK-STAT pathway via phosphorylation of STAT -1 and -2 . The connected activated forms of STAT- 1 and -2 further form complexes with IRF9 with the immediate release of active Interferon stimulating genes (ISGs) resulting in extensive elimination of viral replication [22].

\section{The Adaptive Immune Response}

Humoral immune response, particularly the production of neutralizing antibody, plays an emergency function by restricting the infection at the following stage and prevents re-infection in the future [23]. SARS-CoV-2 infection provokes IgG production against $\mathrm{N}$ protein that can be recognized by serum as early as day 4 after the onset of disease and with most cases seroconverting by day 14 [8]. Laboratory proof of clinical subjects revealed that specific T-cell responses against SARS-CoV-2 are essential for the recognition and killing of infected cells, especially in the lungs of infected people [24]. It has been shown that the number and function of CD8+ T cells were higher than CD4+ T cell responses [4,25], although whether the memory T-cell response is satisfactory to preserve from reinfection needs more investigation [25,26]. Moreover, the virus-specific $\mathrm{T}$ cells from the severe infection tended to have a central memory phenotype with a significantly higher frequency of polyfunctional CD4+ T cells with cytokine secretion, for example, IFN $\gamma, \mathrm{TNF} \alpha$, and IL-2, and CD8+ T cells with cytokine secretion, for example, IFN $\gamma, \mathrm{TNF} \alpha$, and degranulated state, as compared 
with mild infections. Strong T cell responses correlate with higher neutralizing antibody, while higher serum Th2 cytokine secretion, for example, IL-4, IL-5, IL-10 (which progresses the production of antibodies), were diagnosed in the deceased cases 8. Immune system responses after coronavirus infection are shown in Figure 2.

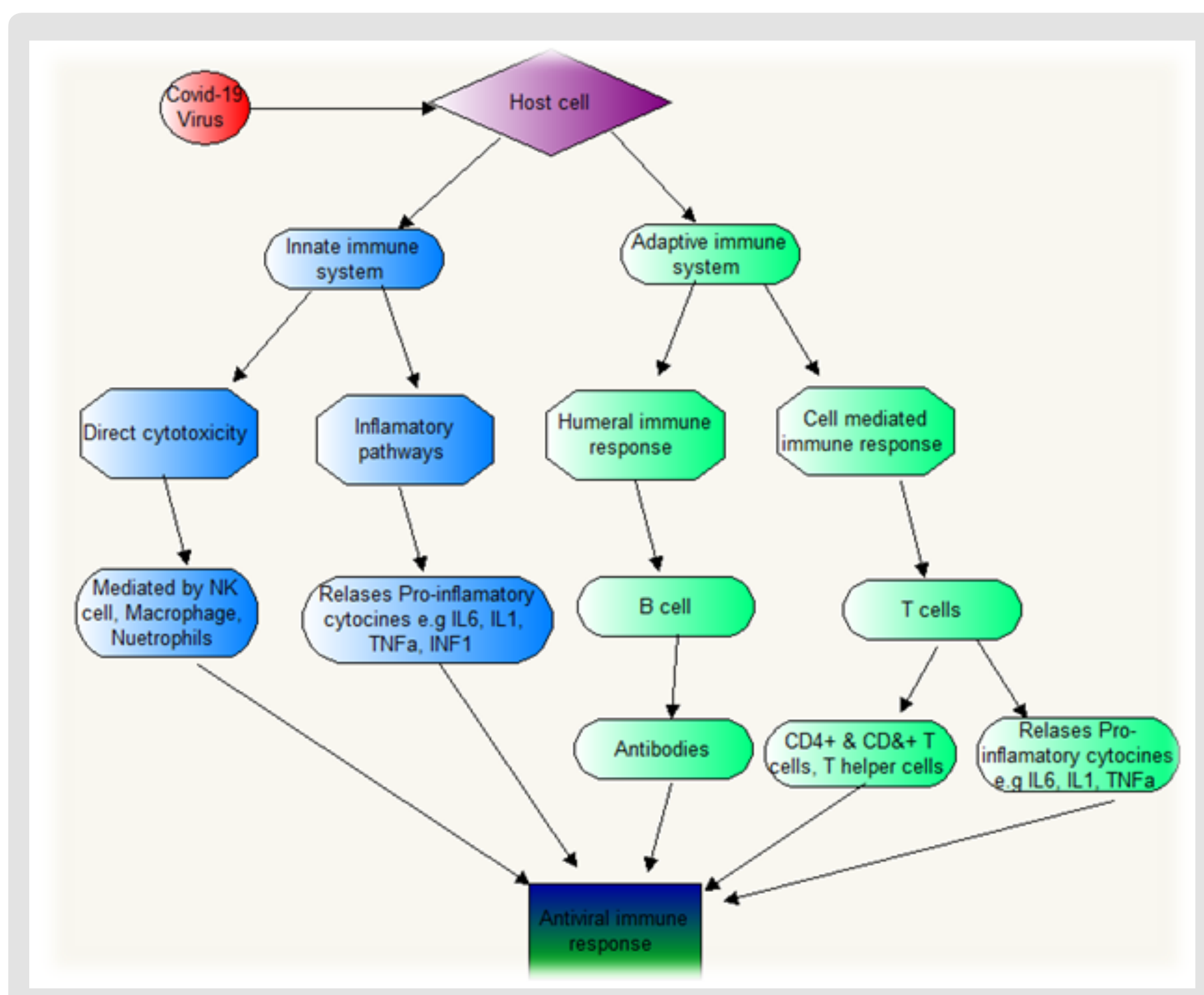

Figure 2: Description of immune system and activation of antiviral immune response to SARS-COV-2.

\section{Exercise and Infection}

In recent years, there have been many researches on exercise immunology, with $90 \%$ of all researches have been carried out since 1990 [27]. The exercise-related adaptation and response of the immune system can be investigated from several aspects. In fact, the immune system responds differently depending on the duration, intensity, acuity, and chronicity of the exercise, which can reduce or increase the risk of infection. In this review study, we intend to discuss the responses of the immune system to exercise according to the variables of intensity, duration, acuity, and chronicity of exercises, and offer suggestions for strengthening the immune system in order to reduce the risk of SARS-COV-2 infection according to the cellular pathways involved in this disease mentioned above. The American College of Sports Medicine (ACSM) divides aerobic activities with moderate to high intensity as follows (Table 1)[28].One of the most important theories of exercise immunology states that exercise can have immunoenhancing and immunosuppressive effects, and this has led researchers to propose J-curve and Open window theories (Figures $3 \& 4$ ). These theories generally state that the best immunoenhancing benefits of exercise are achieved in the case of moderate-intensity activity carried out usually within less than 1.5 hours and the risk of infection is high until hours after exercise [10]. 


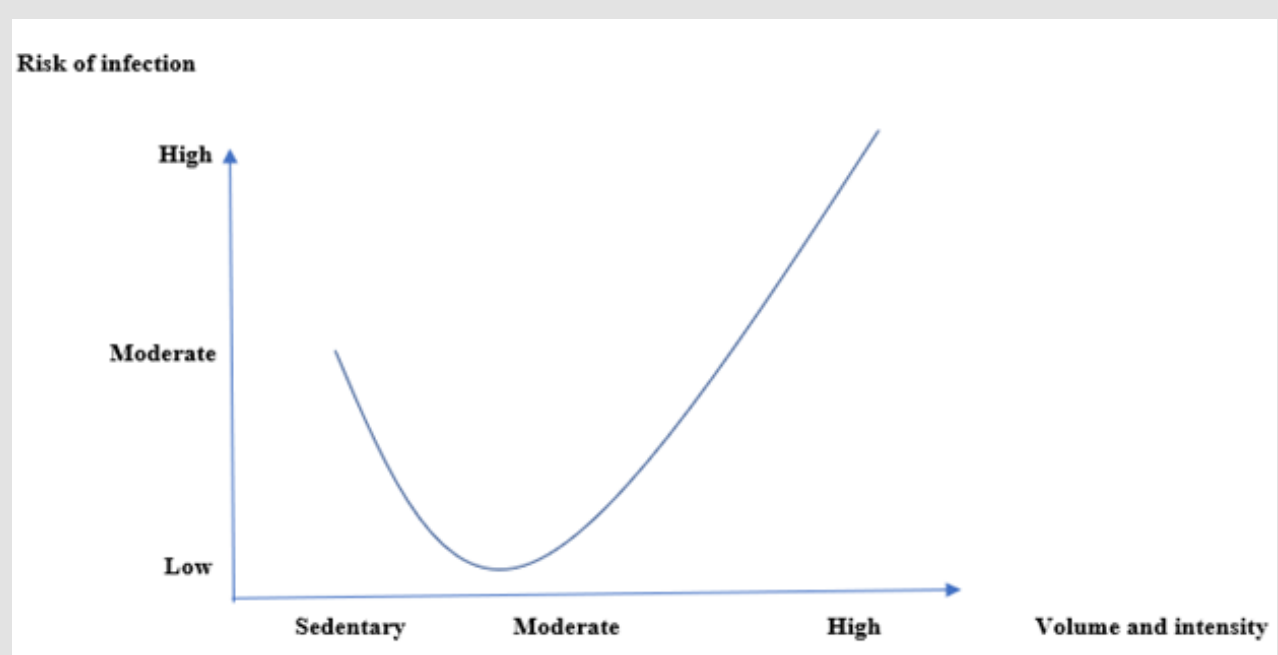

Figure 3: Exercise and risk of infection (J-Curve theory).

\section{Open Window Theory}

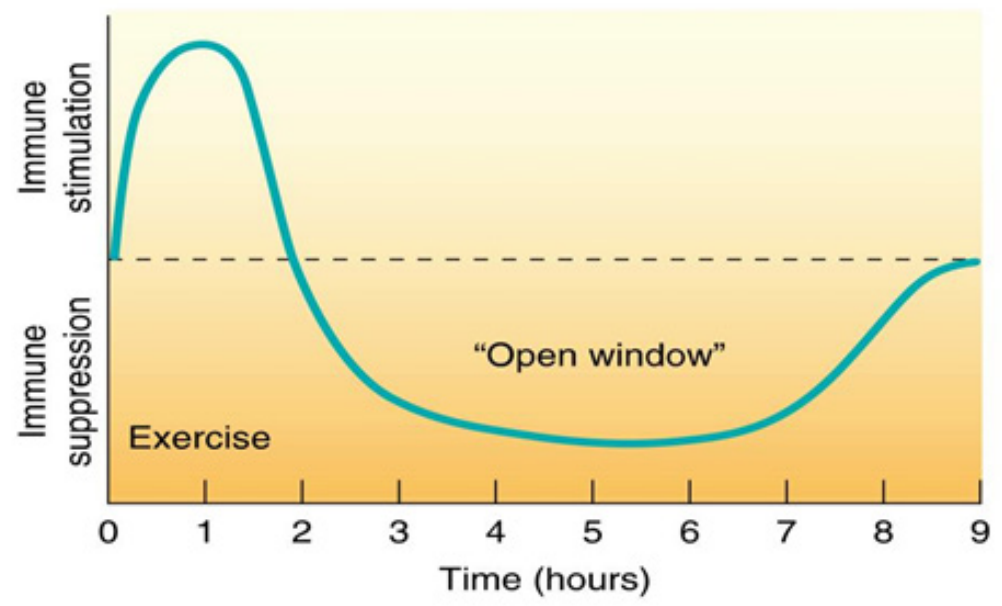

Figure 4: Open window theory.

Table 1: Moderate and Intense aerobic exercise.

\begin{tabular}{|c|c|c|c|c|}
\hline MET & RPE & HR & V02max & Type of Exercise \\
\hline $3-6$ & $12-13$ & $40-60 \% \mathrm{HRR}$ & $40-60 \%$ VO2max & Moderate intensity \\
\hline$>6$ & $14-16$ & $>60 \% \mathrm{HRR}$ & $>60 \%$ V02max & High intensity \\
\hline
\end{tabular}

Note: VO2max: Maximum Rate of Oxygen Consumption; HR: Heart rate; MHR: Maximum Heart Rate; RPE: Rating of Perceived Exertion; MET: Metabolic Equivalent of TaskTerra et al. (2012) reported moderate-intensity exercises synthesize neutrophils and

macrophages, increase IFNy, TNFa activity, and increase the immune system's ability to kill intracellular microorganisms and infections by enhancing the function of Th1 cells, while, intense exercise significantly increases the risk of infection by reducing the chemotactic function of neutrophils, increasing the function of Th2 cells (increasing IL4, IL10, and TGF- $\beta$ ), reducing macrophage activity, apoptosis of lymphocytes, and decreasing TLRs expression
[15]. Natural killer (NK) cells can kill virus-infected cells independently when attacked by viruses [29]. It is well documented that NK cells are very sensitive to the exercise-induced stress, which promotes their redistribution from the peripheral blood to other tissues. Exercise leads to a decrease in the expression of adhesion molecules by inducing catecholamine secretion, and this plays an essential role in the mobilization of peripheral circulation 
of NK cells. However, during excessive prolonged exercise $(>3 \mathrm{~h})$, the concentration of circulating NK cells may return to the preexercise level, or even become lower. It is hypothesized that this decrease is due to the migration of these cells to sites of muscular injury 15. Studies have shown that the CD4+ / CD8+ ratio decreases during exercise, which is due to the significant increase in CD8+ level as compared to CD4+ level during exercise [30]. Lymphocyte concentration decreases significantly after exercise. Researchers have found that this reduction is partly due to elevated apoptotic mechanisms after exercise. When exercises at 38\%,61\%, and $100 \%$ V02max intensities are compared, it is found that apoptotic gene expression levels of lymphocytes increased with increasing exercise intensity from $6.9 \pm 0.5 \%$ (38\% vo2max) to $0.4 \pm 22.4(100 \%$ vo2max) after exercise. Increasing exercise intensity is associated with decreased glutathione (GSH) concentration in lymphocytes, increased oxidative stress, and overexpression of apoptotic factors of Caspase 3, 8, and 9, which can lead to decreased lymphocyte concentration after high-intensity exercise and a decline in lymphocyte ability to defend against infections until it returns to normal $[15,31,32]$. In contrast, some researchers have shown that post-exercise low lymphocyte count may not indicate apoptosis, which is due to the redistributed out-of-blood distribution of the bloodstream to tissue and organs[10]. This phenomenon has been demonstrated in rodents with fluorescent cell tracking and in humans by the proportional reduction of cells responsible for homing receptor expression for tissue and organ sites[33]. On the other hand, salivary Immunoglobulin A (SIgA) measurement may indicate the effects of exercise on humoral immunity. In this regard, several studies have shown that exercise leads to a 20$25 \%$ reduction in sIgA concentration for several hours and have concluded that the risk of infection is high until immunoglobulin A returns to its normal physiological state; however, some research reported no post-exercise reduction in SIgA[10]. SIgA levels vary greatly during different hours of the day and on different days, and individual differences and factors such as oral health, diet, sleep quality, and psychological and physiological stress can also lead to these differences [34]. Therefore, some researchers don't regard the downregulation of SIgA expression as a definite symptom of an increased risk of infection. Another important topic in the field of exercise immunology is the increased risk of infection, especially upper respiratory tract infection (URTI) after high-intensity activities[35]. To justify this, many researchers point to the high rate of URTI in athletes during competitions [36]. On the other hand, it can be stated that factors such as stress caused by competitions, changes in the circadian rhythm of athletes Table 2: Suggested exercise strategies to reduce risk of infection. during competitions, and exposure to infectious agents such as spectators and different places can affect the increase of URTI during competitions. Moreover, this is not necessarily due to the independent effect of exercise, and further research is needed $[10,37]$. Gleason et al. reported that moderate to intense exercises (55-75\% of VO2 max) lasting for more than 1.5 hours lead to a reduction in many functions of the innate immune system, including neutrophil chemotaxis, phagocytosis, decreased TLRs, NK cell activity, and activity of the innate immune system, including the supply of antigens by monocytes/macrophages, immunoglobulin production by B lymphocytes, as well as cytokines produced by $\mathrm{T}$ lymphocytes such as gamma interferon, and thus an increased risk of infection until the recovery of the immune system [38,39]. The incidence of immune system disorders after acute and long-term exercise seem to be due to increased circulating stress hormones (such as epinephrine and norepinephrine) and changes in pro-/ anti-inflammatory cytokine balance 39 . On the other hand, many studies have shown an improvement in immune system function in response to chronic exercises [40-42]. These changes are generally related to increased chemotactic capacity of immune cell and supply of effective antigens against microorganisms, indicating a lower risk of infection in these individuals [39,42]. However, it should be noted that the risk of infection is still high between the end of the training session and the recovery of the immune system. The time it takes to fully recover varies depending on the type of exercise and generally varies from a few hours to one to two days depending on the intensity of the exercise $[43,44]$. Numerous studies have been conducted on the immune system's ability to respond to resistance training exercises[45,46]. The results of studies generally show that resistance exercise leads to a transientperturbations of the immune system, which is in fact reminds us of the open window theory. However, it has been well established that regular resistance training exercises along with a proper recovery period generally reduce systemic inflammation as well as the risk of infection (reference). It should also be noted that the benefits of resistance training exercises ( $<75 \% 1 \mathrm{RM})$ lasting less than 1.5 hours are recommended to achieve different adaptations to improve immune system performance. These adaptations generally include increasing neutrophil count, increasing chemotactic properties of NK cells and neutrophils, improving the response of $\mathrm{T}$ cells against microorganisms, and supplying appropriate antigens and antibodies $[45,47]$. Table 2 demonstrates exercise strategies to prevent infection and increase the immune system's ability to fight microorganisms according to the foregoing.

\begin{tabular}{|c|c|}
\hline \multicolumn{2}{|c|}{ Suggested Exercise Strategies to Reduce Risk of Infection } \\
\hline Frequency & Four to six per week (more sessions with less time are also appropriate) \\
\hline Duration & Less than 1.5 hours (30-45 minutes is very convenient) \\
\hline Intensity & $<70 \%$ V02max in aerobic \\
& $<75 \% 1 \mathrm{RM}$ in resistance training \\
\hline
\end{tabular}




Load (volume*intensity) $\quad$ Systematically manipulate either the training volume and/or intensity to manage the degree of training load
Recovery The immune system usually needs to 8 hours to recover after exercise. During this time, follow the hygiene tips.

\section{Conclusion}

The aim of the present study was to provide reliable exercise strategies for healthy individuals to strengthen the immune system and prevent infection and SARS-COV-2. As we have shown in this study, there are strong evidences that chronic moderate-intensity exercise has immune-enhancing effects and reduces the risk of infection through a variety of mechanisms. On the other hand, the effects of acute and high-intensity exercise on the immune system are still one of the most controversial topics in exercise immunology. After acute and high-intensity exercise, the risk of infection seems to increase until recovery of the immune system, which is mainly due to the response of the immune system to muscle and cell damage after these exercises. Therefore, considering the limited evidence, such exercises are not recommended in the current situation to prevent infection. However, further research is needed to make a definitive statement. Future researches on animal models could prove the impact of different types of exercise with different intensities and durations on the risk of various infections, including SARS-COV-2. It can also be helpful to study effective strategies to prevent infection during the open window and speed up the recovery process of the immune system after exercise. Finally, it can be stated that exercise (considering the foregoing) can probably play a preventive role against various infections, including SARS$\mathrm{COV}-2$, and considering that no vaccine has yet been discovered to treat this disease, it is hoped that this will prevent the high rate this infection among healthy people.

\section{Acknowledgment}

There was no financial support for this study

\section{Authors' Contribution}

M.K and M.P wrote the manuscript. All authors have read and approved the final version of the manuscript and agree with the order of the presentation of the authors.

\section{Competing Interest}

No potential conflict of interest was reported by the authors.

\section{References}

1. Raoult D, Zumla A, Locatelli F, Ippolito G, Kroemer G (2020) Coronavirus infections: Epidemiological, clinical and immunological features and hypotheses. Cell stress 4(4): 66.

2. Lai CC, TP Shih, WC Ko, HJ Tang, PR Hsueh (2020) Severe acute respiratory syndrome coronavirus 2 (SARS-CoV-2) and corona virus disease-2019 (COVID-19): the epidemic and the challenges. International journal of antimicrobial agents :105924.

3. (2020) WHO. Coronavirus disease (COVID-19) Situation Report-188.

4. Li G, Y Fan, Y Lai, T Han, Z Li, et al. (2020) Coronavirus infections and immune responses. Journal of medical virology 92(4): 424-432.

5. Walls AC, YJPark, MA Tortorici, A Wall, AT Mc Guire, et al. (2020) Structure, function, and antigenicity of the SARS-CoV-2 spike glycoprotein. Cell.
6. Yan R, Y Zhang, Y Li, L Xia, Y Guo, et al. (2020) Structural basis for the recognition of SARS-CoV-2 by full-length human ACE2. Science 367(6485): 1444-1448.

7. Weiss SR, S Navas-Martin (2005) Coronavirus pathogenesis and the emerging pathogen severe acute respiratory syndrome coronavirus. Microbiol Mol Biol Rev 69(4): 635-664.

8. Rokni M, V Ghasemi, Z Tavakoli (2020) Immune responses and pathogenesis of SARS-CoV-2 during an outbreak in Iran: Comparison with SARS and MERS. Reviews in Medical Virology.

9. Sheshe S, A Nazifi, A Labbo, G Khalid, A Yahya, et al. (2020) Mechanism of Antiviral Immune Response and COVID-19 Infection. Asian Journal of Immunology :1-8.

10. Simpson RJ, JP Campbell, M Gleeson, K Krüger, DC Nieman, et al. (2020) Can exercise affect immune function to increase susceptibility to infection? Exercise Immunology Review 26: 8-22.

11. Martin SA, BD Pence, JA Woods (2009) Exercise and respiratory tract viral infections. Exercise and sport sciences reviews 37(4): 157.

12. Harris MD (2011) Infectious disease in athletes. Current sports medicine reports 10(2): 84-89.

13. (2020) WHO. Coronavirus disease (COVID-2019) situation reports; 2020

14. Ortega E (2016) The "bioregulatory effect of exercise" on the innate/ inflammatory responses. Journal of physiology and biochemistry $72(2)$ : 361-369.

15. Terra R, SAGd Silva, VS Pinto, PML Dutra (2012) Efeito do exercício no sistema imune: resposta, adaptação e sinalização celular. Revista Brasileira de Medicina do Esporte 18: 208-214.

16. Nürnberger T, B Kemmerling (2018) Pathogen-Associated Molecular Patterns (PAMP) and PAMP-Triggered Immunity. Annual Plant Reviews online: $16-47$

17. De Wit E, N Van Doremalen, D Falzarano, VJ Munster (2016) SARS and MERS: recent insights into emerging coronaviruses. Nature Reviews Microbiology 14(8): 523

18. Channappanavar R, S Perlman (2017) Pathogenic human coronavirus infections: causes and consequences of cytokine storm and immunopathology. Seminars in immunopathology. 39. Springer: 529539.

19. Yang CH, K Li, SR Pfeffer, LM Pfeffer (2015) The type I IFN-induced miRNA, miR-21. Pharmaceuticals 8(4): 836-847.

20. Kindler E, V Thiel, F Weber (2016) Interaction of SARS and MERS coronaviruses with the antiviral interferon response. Advances in virus research. Elsevier: 219-243.

21. Prompetchara E, C Ketloy, T Palaga (2020) Immune responses in COVID-19 and potential vaccines: Lessons learned from SARS and MERS epidemic. Asian Pac J Allergy Immunol 38(1): 1-9.

22. Song Z, Y Xu, L Bao, L Zhang, P Yu, et al. (2019) From SARS to MERS, thrusting coronaviruses into the spotlight. Viruses 11(1): 59.

23. Davies DH, X Liang, JE Hernandez, A Randall, S Hirst, et al. (2005) Profiling the humoral immune response to infection by using proteome microarrays: high-throughput vaccine and diagnostic antigen discovery. Proceedings of the National Academy of Sciences 102(3): 547-552.

24. Wang X, W Xu, G Hu, S Xia, Z Sun, et al. (2020) SARS-CoV-2 infects T lymphocytes through its spike protein-mediated membrane fusion. Cellular \& Molecular Immunology :1-3.

25. Channappanavar R, C Fett, J Zhao, DK Meyerholz, S Perlman (2014) Virus-specific memory CD8 $\mathrm{T}$ cells provide substantial protection from 
lethal severe acute respiratory syndrome coronavirus infection. Journal of virology 88(19): 11034-11044.

26. Chen J, YF Lau, EW Lamirande, CD Paddock, JH Bartlett, et al.(2010) Cellular immune responses to severe acute respiratory syndrome coronavirus (SARS-CoV) infection in senescent BALB/c mice: CD4+ T cells are important in control of SARS-CoV infection. Journal of virology 84(3): 1289-1301.

27. Van Dijk JG, KD Matson (2016) Ecological immunology through the lens of exercise immunology: new perspective on the links between physical activity and immune function and disease susceptibility in wild animals. Integrative and comparative biology 56(2): 290-303.

28. Riebe D, BA Franklin, PD Thompson, CE Garber, GP Whitfield, et al. (2015) Updating ACSM's recommendations for exercise preparticipation health screening. Medicine \& Science in Sports \& Exercise 47(11): 2473-2479.

29. Gyurova IE, Ali A, SN Waggoner (2019) Natural Killer Cell Regulation of B Cell Responses in the Context of Viral Infection. Viral Immunology.

30. Timmons BW, T Cieslak (2008) Human natural killer cell subsets and acute exercise: a brief review. Exerc Immunol Rev 14(905): 8-23.

31. Levada Pires AC, MF Cury-Boaventura, R Gorjao, SM Hirabara, EF Puggina, et al. (2008) Neutrophil death induced by a triathlon competition in elite athletes. Medicine and science in sports and exercise 40(8): 1447-1454.

32. Navalta J, D Sedlock, KS Park (2007) Effect of exercise intensity on exercise-induced lymphocyte apoptosis. International journal of sports medicine 28(06): 539-542.

33. Krüger K, A Lechtermann, M Fobker, K Völker, F Mooren (2008) Exerciseinduced redistribution of $\mathrm{T}$ lymphocytes are regulated by adrenergic mechanisms. Brain, behavior, and immunity 22(3): 324-338.

34. Pritchard BT, W Stanton, R Lord, P Petocz, GJ Pepping (2017) Factors affecting measurement of salivary cortisol and secretory immunoglobulin A in field studies of athletes. Frontiers in endocrinology 8:168.

35. Gleeson M, DB Pyne (2016) Respiratory inflammation and infections in high-performance athletes. Immunology and cell biology 94(2): 124131.

ISSN: 2574-1241

DOI: 10.26717/BJSTR.2020.29.004799

Mohsen Parvani. Biomed J Sci \& Tech Res

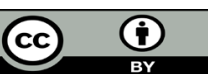

This work is licensed under Creative Commons Attribution 4.0 License

Submission Link: https://biomedres.us/submit-manuscript.php
36. Ahmadi S, C Brietzke, R Silveira, RCR Do Prado, R Brietzke, et al. (2019) Aspects of physical training related with upper respiratory tract infections: a review. Manual Therapy, Posturology \& Rehabilitation Journal 16.

37. Herrero H (2020) Sports and Infections. Injury and Health Risk Management in Sports. Springer 281-286.

38. Gleeson M (2013) Exercise, nutrition and immunity. Diet, immunity and inflammation. Elsevier 652-685.

39. Gleeson M (2015) Effects of exercise on immune function. Sports Sci Exch 28: 1-6.

40. Peake J (2020) Interrelations between acute and chronic exercise stress and the immune and endocrine systems. Endocrinology of physical activity and sport. Springer 249-266.

41.Zimmer P, A Schenk, M Kieven, M Holthaus, J Lehmann, et al. (2017) Exercise induced alterations in NK-cell cytotoxicity-methodological issues and future perspectives. Exercise immunology review 23.

42. Jee YS (2020) How much exercise do we need to improve our immune system? second series of scientific evidence. Journal of Exercise Rehabilitation 16(2): 113-114

43. Peake JM, O Neubauer, NP Walsh, RJ Simpson (2017) Recovery of the immune system after exercise. Journal of Applied Physiology 122(5): 1077-1087.

44. Peake JM (2019) Recovery after exercise: what is the current state of play? Current Opinion in Physiology 10: 17-26.

45. Freidenreich DJ, JS Volek (2012) Immune responses to resistance exercise. Exercise immunology review 18.

46. Spiering BA, WJ Kraemer, JM Anderson, LE Armstrong, BC Nindl, et al (2008) Resistance exercise biology. Sports Medicine 38(7): 527-540

47. Koch AJ (2010) Immune response to resistance exercise. American Journal of Lifestyle Medicine 4(3): 244-252.

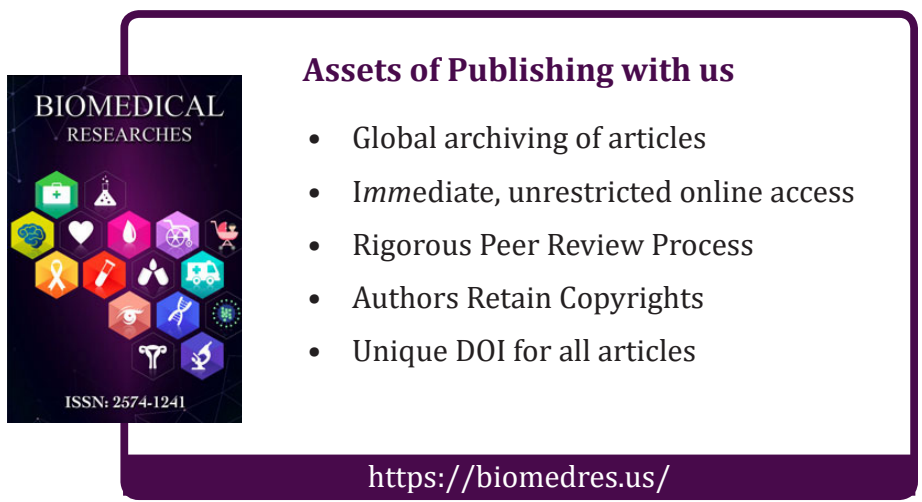

